\title{
Hochdosierte SLIT bei Kindern wirksam und sicher
}

Die sublinguale Immuntherapie (SLIT) stellt insbesondere bei Kindern eine Alternative zu subkutanen Injektionen dar. Prof. Dr. Ulrich Wahn, Berlin, hat die Wirksamkeit bei Kindern mit Gräserpollenallergie untersucht.

n die placebokontrollierte, randomisierte Doppelblindstudie, die an 34 Zentren in Deutschland und Polen durchgeführt worden war, nahmen die Berliner Allergologen 207 Kinder zwischen vier und zwölf Jahren mit allergischer Rhinitis und Rhinokonjunktivitis auf, einige von ihnen auch mit Asthmasymptomatik. Nach der Erstbeobachtung zwischen Februar und Juni erhielten die Probanden von Januar bis August des darauffolgenden Jahres entweder einen hochdosierten (Erhaltungsdosis $40 \mu \mathrm{g} / \mathrm{d}$ ), wässrigen 6-Gräser-Pollenextrakt sublingual (3.600-4.800 $\mu \mathrm{g}$ bis zum Beginn der Graspollensaison und $3.600 \mu \mathrm{g}$ von Mai bis Juli) oder Placebo. Symptome und Medikamentenverbrauch wurden zehn Tage vor dem jeweiligen Pollenflug-Maximum und bis 31 Tage danach täglich beurteilt. Primärer Endpunkt war die Veränderung im Symptom-Medikations-Score (SMS) nach einer Behandlungsperiode im Vergleich zum Ausgangswert. Dabei wurden Au- gen-, Nasen- und Bronchialsymptome bewertet und Häufigkeit sowie Dosierung von Medikamenten berücksichtigt.

Nach der ersten prä- und kosaisonalen Behandlungsperiode war in der Verumgruppe eine Verbesserung des SMS von 558,0 auf 341,8 zu beobachten, in der Placebogruppe von 486,4 auf 402,5. Der Unterschied zwischen Verum und Placebo erwies sich als signifikant. Wurden nur die Ergebnisse von Nasen- und Augensymptomen berücksichtigt, ergab sich für die Verumgruppe eine ebenfalls signifikante Symptomreduktion um 199,7 Score-Punkte vs. 83,6 mit Placebo. Die Effekte bestätigten sich auch bei der Einzelbetrachtung von Symptom- und Medikations-Score. Die Anzahl der Tage, an denen die Probanden eine gute Symptomkontrolle erreichten, nahm unter SLIT ebenfalls zu. Gleichzeitig war gegenüber Placebo eine deutliche Immunreaktion zu beobachten, die sich durch den Anstieg der IgG1- und IgG4-Spiegel nachweisen ließ. Trotz hoher Dosierung

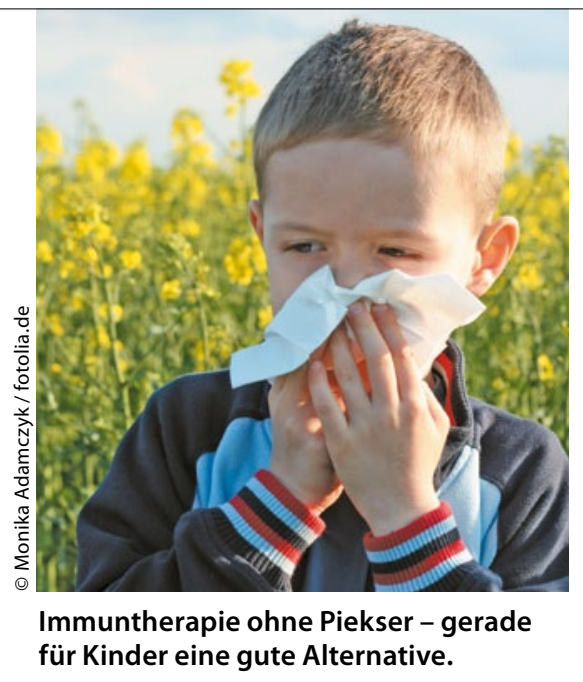

wurde die SLIT gut vertragen. 71,5 \% der Verumpatienten berichteten über lokale Störungen am Applikationsort vs. 12,2 \% der Kontrollgruppe.

Fazit: Die tägliche Gabe einer hochdosierten SLIT ist bei einer Gräserpollenallergie wirksam und wird von Kindern gut vertragen. Bei 50,8\% der Probanden verbesserte sich der Symptom- und Medikations-Score innerhalb einer Behandlungsperiode um mindestens $40 \%$. In der Placebogruppe um 33,3\%. Dr. Christine Starostzik

Wahn U. et al. High-dose sublingual immunotherapy with single-dose aqueous grass pollen extract in children is effective and safe: A double-blind, placebo-controlled study. J Allergy Clin Immunl 2012; 130: 886-93

\section{Verquollene Augen - ein unterschätztes Symptom}

Es ist gar nicht so sehr das Niesen, was Allergiegeplagten zu schaffen macht. Am lästigsten ist, wenn man zwischen geschwollenen Lidern kaum mehr aus den Augen schauen kann, aber dagegen wird nur selten etwas unternommen.

ranzösische Forscher haben 980 Pati- enten, die wegen ihrer allergischen Rhinitis eine Allgemeinarztpraxis aufgesucht hatten, nach ihren Beschwerden und deren Einfluss auf die Lebensqualität befragt. Folgende Symptome wurden erfasst: Niesen, eine laufende, juckende oder verstopfte Nase, Augensymptome und juckende Ohren.

Bindehautentzündung und geschwollene Lider waren eindeutig am lästigsten. Wer deswegen buchstäblich kaum mehr aus den Augen schauen konnte, fühlte sich dreimal mehr in der Lebensqualität beeinträchtigt als Patienten ohne Augensymptome. Was als unangenehm emp- funden wurde, war auch die körperliche Schlappheit. Eine verstopfte Nase hatte dagegen überraschend wenig Einfluss auf die Lebensqualität, die laufende Nase und das Niesen gar keinen. Von allen Symptomen war die Rhinorrhö am häufigsten (89\%), gefolgt von Niesen und verstopfter Nase (jeweils $82 \%$ ), nasalem Juckreiz und Augensymptomen (jeweils 68\%). Über $60 \%$ der Patienten litten unter mäßigen bis schweren Symptomen.

Was die Forscher besonders erstaunte: Die Therapie (hier kamen z. B. Antihistaminika, Kortison und abschwellende Mittel zum Einsatz) hatte offenbar kaum Auswirkungen auf die Schwere der Sym- ptome und das Wohlbefinden der Patienten. Das zeigte ein Test mit einer visuellen Analogskala. Unbehandelte Patienten gaben auf der von $0 \mathrm{~mm}$ (gar nicht störend) bis $100 \mathrm{~mm}$ (unerträglich) reichenden Skala eine Symptomstärke von rund 61 an, behandelte Patienten ebenso!

Fazit: Die systemische Therapie mit Antihistaminika, so die HNO-Experten um Prof. Dr. Philippe Jean Bousquet, Montpellier, führt v.a. dazu, dass sich die nasalen Symptome bessern; an den Augen ist der Erfolg dagegen oft bescheiden. Generell gebe es bei der Therapie von Augensymptomen erheblichen Nachholbedarf.

Dr. Elke Oberhofer

Bousquet PJ et al. Impact of Allergic Rhinitis Symptoms on Quality of Life in Primary Care. Int Arch Allergy Immunol 2013; 160: 393-400 